\title{
EL USO DE MATRICES DAFO COMO HERRAMIENTAS DE GESTIÓN Y ANÁLISIS GEOGRÁFICO.
}

Dra. Ana María Foschiatti (UNNE-CONICET)

Mg. Jorge Alfredo Alberto (UNNE)

Av. Las Heras 727 - 3500 Resistencia (Chaco -Argentina)

amfoschiatti@hum.unne.edu.ar

jaalberto@hum.unne.edu.ar

Resumen

El análisis DAFO es una herramienta sencilla de utilizar, pero muy potente como mecanismo de análisis de la realidad y de la toma de decisiones. Su nombre proviene de las cuatro ideas que centran el mismo: Debilidades, Amenazas, Fortalezas y Oportunidades. Es una metodología de estudio donde se analiza la situación externa e interna y cuyo objetivo es determinar las características de un escenario. Se puede denominar también con las expresiones FODA, DOFA y AODF. Su principal objetivo consiste en sintetizar, en un gráfico o tabla resumen, la valoración de las cuestiones fuertes y débiles y de las amenazas y oportunidades externas, coherentes con la estrategia hacia el logro de un adecuado ajuste entre su capacidad interna y su posición externa

Palabras claves: DAFO; Diagnóstico; Metodología

\section{THE USE OF SWOT ARRAYS AS TOOLS FOR GEOGRAPHIC ANALYSIS AND MANAGEMENT}

Abstract

The SWOT analysis is a simple tool to use, and a very powerful reality analysis mechanism for taking decisions. Its name comes from four ideas that it focuses: Weaknesses, Threats, Fortresses and Opportunities. It is a study methodology which analyses external and inner situations, whose aim is to determine the characteristics of a stage. It can be also designated with the expressions FODA, DOFA and AODF. Its main goal consists on synthesize in a chart or summary table, the assessment of strong and feeble issues, and the evaluation of the external threats and opportunities, consistent with a strategy towards achieving a proper fit between its internal capacity and its external position

Keywords: SWOT; Diagnose; Methodology 


\section{INTRODUCCIÓN}

El Análisis FODA, AODF o DAFO (Debilidades Amenazas, Fortalezas y Oportunidades) o SWOT (Strengths, Weakness, Oportunities and Threats) es una metodología de estudio donde se analiza la situación externa e interna cuyo objetivo es determinar las características de un escenario.

Consiste en sintetizar, identificar y medir, en un gráfico o tabla resumen, la valoración de las cuestiones fuertes - débiles y de las amenazas - oportunidades externas, en coherencia con la lógica de que la estrategia debe lograr un adecuado ajuste entre su capacidad interna y su posición externa.

Las fortalezas y debilidades internas resultan importantes puesto que pueden ayudarnos a entender la posición del problema en un entorno concreto. Un primer paso, por tanto, consiste en analizar el ambiente, es decir cuáles son las variables (factores críticos de éxito -en adelante FCE-) apropiadas a utilizar.

Una vez definidos los FCE se debe realizar un proceso de benchmarking o análisis comparativo. Este proceso puede llevar incluso a identificar nuevas oportunidades.

La situación interna se compone de dos factores controlables (fortalezas y debilidades), mientras que la situación externa se compone de dos factores no controlables (oportunidades y amenazas).

Este recurso fue creado a principios de la década de los setenta y produjo una revolución en el área de la dirección estratégica de una empresa. Su objetivo es determinar las ventajas competitivas de la misma y la estrategia a emplear que más le convenga en función de las características propias y del mercado en que se mueve. En este caso intentamos utilizar su metodología al estudio de situaciones sociales y demográficas (Cfr. Dyson, 2004). Asimismo puede definirse como la herramienta estratégica más utilizada por excelencia para conocer la situación real en que se encuentra una organización.

El análisis consta de cuatro pasos:

- ANÁLISIS EXTERNO (También conocido como "Modelo de las cinco fuerzas se Porter")

- ANÁLISIS INTERNO

- CONFECCIÓN DE LA MATRIZ DAFO

- EVALUACIÓN DE LA ESTRATEgIA A EMPLEAR

\section{ANÁLISIS EXTERNO}

Las situaciones sociales y demográficas no existen ni pueden existir fuera de un ambiente ni de un entorno, por ello el análisis externo permitirá fijar las oportunidades y amenazas que el contexto puede presentarle a esa población. El proceso para determinar esas oportunidades o amenazas se puede realizar de la siguiente manera:

a. Determinar los principales hechos o eventos del ambiente que tienen alguna relación con las situaciones analizadas. Estos pueden subdividirse de acuerdo a su naturaleza (Tabla 1).

\section{Tabla 1. Naturaleza de los eventos}

\begin{tabular}{|l|l|}
\hline Naturaleza del Evento & Evento \\
\hline Político & $\begin{array}{l}\text { Estabilidad política del país } \\
\text { Sistema de gobierno } \\
\text { Relaciones internacionales } \\
\text { Restricciones migratorias }\end{array}$ \\
\hline \multirow{2}{*}{ Legal } & $\begin{array}{l}\text { 1. Tendencias fiscales } \\
\text { Presión impositiva } \\
\text { Pago de impuestos }\end{array}$ \\
\hline
\end{tabular}




\begin{tabular}{|l|l|}
\hline \multirow{5}{*}{} & $\begin{array}{l}\text { 2. Legislación } \\
\text { Laboral } \\
\text { Mejoramiento del ambiente } \\
\text { Descentralización de las zonas urbanas } \\
\\
\text { 3. Económicas } \\
\text { Nivel de salarios } \\
\text { Nivel de precios } \\
\text { Inversiones }\end{array}$ \\
\hline Social & Crecimiento y distribución demográfica \\
& Empleo y desempleo \\
& Sistema de salubridad e higiene \\
\hline \multirow{2}{*}{ Tecnológico } & Rapidez de los avances tecnológicos. \\
& Cambios en los sistemas. \\
\hline
\end{tabular}

Fuente: DYSON, ROBERT G. (2004)

b. Determinar cuáles son los factores que podrían tener influencia sobre la organización en términos de facilitar o restringir el logro de los objetivos. En ese sentido hay circunstancias o hechos presentes en el ambiente que a veces representan una buena OPORTUNIDAD para aprovechar en la resolución de problemas. También puede haber situaciones que más bien representan AMENAZAS y que pueden agravar los problemas.

Las OPORTUNIDADES son las situaciones externas positivas, que se originan en el entorno y que pueden ser aprovechadas. Algunos de los interrogantes que ayudan a su desarrollo son:

¿A qué tipo de oportunidades se enfrenta la sociedad?

¿Qué situaciones de coyuntura económica colaborarán en el desarrollo social?

¿Influyen los cambios tecnológicos en el mejoramiento de la calidad de vida?

¿Qué papel cumplen las normativas legales y la política en la sociedad?

¿Qué cambios en los patrones sociales y de estilos de vida se están presentando?

Las AMENAZAS son situaciones externas negativas, que pueden atentar contra el entorno, por lo que dadas las circunstancias, será necesario diseñar estrategias adecuadas para poder sortearlas. Algunas de las preguntas que contribuyen en su desarrollo son:

¿Cuáles son los obstáculos se deberán enfrentar?

¿Qué influencia ejercen las acciones políticas y sociales sobre el medio?

¿Pueden las amenazas impedir totalmente las actividades de las personas?

\section{ANÁLISIS INTERNO}

Los elementos internos que se deben analizar durante el proceso de análisis DAFO corresponden a las fortalezas y debilidades que se tienen respecto a la disponibilidad de recursos humanos, activos, calidad de vida, estructura urbana, percepción social, entre otros.

El análisis interno fija las FORTALEZAS y DEBILIDADES de la sociedad, mediante un estudio que permite conocer la cantidad y calidad de los recursos y procesos con que se cuenta.

Para realizar el análisis interno debe aplicarse diferentes técnicas que permitan identificar los atributos que generan situaciones ventajosas.

Las FORTALEZAS son todos aquellos elementos internos positivos que ofrecen ventajas a la sociedad. Algunas de las preguntas que se realizan y contribuyen en su desarrollo son:

¿Qué ventajas se obtienen con su aplicación? 
¿Qué hace la sociedad para mejorar?

¿A qué recursos se tiene acceso?

Contrariamente, las DEBILIDADES se refieren a todos aquellos elementos, recursos, habilidades y actitudes negativas que constituyen barreras para el logro de los objetivos. Las Debilidades son problemas internos, que una vez identificadas y aplicando una adecuada estrategia deberían ser eliminadas. Algunas de las preguntas que se pueden realizar y que contribuyen en el desarrollo son:

¿Qué se puede mejorar?

¿Que se debería evitar?

¿Qué factores reducen el éxito de un proyecto?

\section{CONFECCIÓN DE LA MATRIZ DAFO}

De la combinación de las fortalezas y las oportunidades surgen las fuerzas que señalan el camino para delinear acciones importantes, y la combinación de las debilidades y las amenazas se limitan y advierten sobre los problemas (Dyson 2004).

Mientras que los riesgos (fortalezas y amenazas) y los desafíos (debilidades y oportunidades), con su correspondiente combinación de factores, exigirán una cuidadosa consideración a la hora de tomar decisiones para asumir el futuro deseable.

La matriz DAFO se confeccionó teniendo en cuenta el análisis interno y externo, como así las fortalezas, debilidades, oportunidades y amenazas:

\section{Tabla 2. Matriz DAFO simple}

\begin{tabular}{|c|l|l|}
\hline \multirow{2}{*}{ Análisis Interno } & \multicolumn{1}{|c|}{ FORTALEZAS } & \multicolumn{1}{c|}{ DEBILIDADES } \\
\cline { 2 - 3 } & $\begin{array}{l}\text { Capacidades distintas } \\
\text { Ventajas Naturales } \\
\text { Recursos superiores }\end{array}$ & $\begin{array}{l}\text { Recursos y capacidades escasas } \\
\text { Resistencia al cambio } \\
\text { Problemas de motivación }\end{array}$ \\
\hline \multirow{3}{*}{ Análisis Externo } & \multicolumn{1}{|c|}{ OPORTUNIDADES } & \multicolumn{1}{c|}{ AMENAZAS } \\
\cline { 2 - 3 } & $\begin{array}{l}\text { Nuevas tecnologías } \\
\text { Debilitamiento social } \\
\text { Posicionamiento estratégico }\end{array}$ & $\begin{array}{l}\text { Altos riesgos } \\
\text { Cambio en el entorno }\end{array}$ \\
\hline
\end{tabular}

Fuente: DYSON, ROBERT G. (2004)

\section{LA PLANIFICACIÓN ESTRATÉGICA}

Durante la etapa de planificación estratégica utilizando el análisis DAFO se pretendió argumentar cada una de las siguientes preguntas:

¿Cómo se puede explotar cada fortaleza?

¿Cómo se puede aprovechar cada oportunidad?

¿Cómo se puede detener cada debilidad?

¿Cómo se puede defender de cada amenaza?

Según Fred R. David (1997) la matriz amenazas-oportunidades-debilidades-fuerzas (AODF) es un instrumento de ajuste fundamental que ayuda a explicar cuatro tipos de estrategias: estrategias de fuerzas y oportunidades (FO), estrategias de debilidades y oportunidades (DO), estrategias de fuerzas y amenazas (FA) y estrategias de debilidades y amenazas (DA). Observar los factores internos y externos clave es la parte más compleja para desarrollar una matriz DAFO se requieren criterios firmes para presentarla. Tabla 3. 
Las estrategias FO aprovechan las fuerzas internas para usar las ventajas de las oportunidades externas. Por lo general, las organizaciones siguen a las estrategias de DO, FA o DA para colocarse en una situación donde se puedan aplicar estrategias FO. Cuando una sociedad tiene debilidades importantes, luchará por superarlas y convertirlas en fuerzas. Cuando una organización enfrenta amenazas importantes, tratará de evitarlas para fortalecerse en las oportunidades. (David 1997)

Las estrategias DO intentan superar las debilidades internas valiéndose de las oportunidades externas. En ocasiones existen oportunidades externas clave, pero pueden manifestarse debilidades internas que imposibilitan aprovechar dichas oportunidades.

Las estrategias FA utilizan las fuerzas para prescindir o reducir las consecuencias de las amenazas externas. Esto no significa que una buena organización deba enfrentar las amenazas del escenario externo.

Las estrategias DA son procedimientos defensivos que intentan reducir las debilidades internas y remediar las amenazas del ambiente. Una sociedad que enfrenta amenazas externas y debilidades internas podría estar en una situación muy precaria.

La siguiente tabla esquematiza una matriz DAFO para la formulación de estrategias.

Tabla 3. Matriz DAFO para la formulación de estrategias

\begin{tabular}{|l|l|l|}
\hline Dejar en blanco & $\begin{array}{l}\text { FUERZAS-F } \\
\text { Anotar las fuerzas }\end{array}$ & $\begin{array}{l}\text { DEBILIDADES-D } \\
\text { Anotar las debilidades }\end{array}$ \\
\hline $\begin{array}{l}\text { OPORTUNIDADES-O } \\
\text { Anotar las oportunidades }\end{array}$ & $\begin{array}{l}\text { ESTRATEGIAS-FO } \\
\text { Usar las fuerzas para } \\
\text { aprovechar las oportunidades }\end{array}$ & $\begin{array}{l}\text { ESTRATEGIAS-DO } \\
\text { Superar las debilidades } \\
\text { aprovechando las oportunidades }\end{array}$ \\
\hline $\begin{array}{l}\text { AMENAZAS-A } \\
\text { Anotar las amenazas. }\end{array}$ & $\begin{array}{l}\text { ESTRATEGIAS-FA } \\
\text { Usar las fuerzas para evitar las } \\
\text { amenazas }\end{array}$ & $\begin{array}{l}\text { ESTRATEGIAS-DA } \\
\text { Reducir las debilidades y evitar } \\
\text { las amenazas }\end{array}$ \\
\hline
\end{tabular}

Fuente: David, Fred (1997)

La primera, segunda, tercera, y cuarta estrategia son: FO, DO, FA, y DA, respectivamente.

En la matriz DAFO precedente se observan nueve celdas. Hay cuatro para factores clave, cuatro para estrategias y una celda que siempre se deja en blanco (la superior de la izquierda). Las cuatro celdas de las estrategias llamadas FO, DO, FA, DA se ocupan después de llenar las cuatro de los factores claves, llamados F, D, O, A. La matriz DAFO se lleva a cabo siguiendo los ocho pasos siguientes (David 1997):

- Hacer una lista de las oportunidades externas.

- Hacer una lista de las amenazas externas.

- Hacer una lista de las fuerzas internas.

- Hacer una lista de las debilidades internas.

Adecuar las fuerzas internas a las oportunidades externas y registrar las estrategias FO resultantes en la celda adecuada.

Adecuar las debilidades internas a las oportunidades externas y registrar las estrategias DO resultantes en la celda adecuada.

Adecuar las fuerzas internas a las amenazas externas y registrar las estrategias FA resultantes en la celda adecuada.

Adecuar las debilidades internas a las amenazas externas y registrar las estrategias DA resultantes en la celda adecuada. 
Este modelo difundido a nivel mundial, aunque desconocido en muchos ámbitos puede presentar algunas falencias o descuidos en su aplicación (Amaya Correa 2004). Algunos pueden definirse así:

En muchas ocasiones no se tiene claridad sobre el problema o problemas a analizar.

Antes de iniciar un análisis y de determinar el método, se debe tener en cuenta que es más importante identificar su cabalidad para que todos los participantes sepan cual es el problema a analizar.

Debido a la falta de identificación o delimitación del problema en muchas ocasiones el método DAFO puede fracasar, o resultar una pérdida de tiempo.

La identificación del problema debe ser muy precisa, limitando su entorno, sus causas y consecuencias para no alejarse de las circunstancias que no forman parte del problema central o que no son relevantes.

Cuando en el grupo no se tiene conciencia del trabajo y de lo que representa realmente una debilidad, una oportunidad, una fortaleza o una amenaza, es posible que se genere un "torbellino de ideas" que solo provoca una desviación en el tratamiento de los temas centrales. La presencia de personas que no se sienten involucradas en el problema no prestarán colaboración tanto en el diagnóstico como en la solución de los problemas.

En la configuración de la matriz, que concentra el 10\% del proceso DAFO, se tratará de diagnosticar las soluciones a los problemas planteados. Cuando no se sigue trabajando sobre todos los componentes de la matriz: no se plantean estrategias de trabajo sobre cada uno de los puntos presentados, no se toma la molestia de validar los problemas en cada una de las ideas plasmadas en la matriz y por lo tanto no se llegará a la posible solución del mismo. Cuando no se valora el efecto de cada uno de los componentes de la matriz para determinar si realmente todos son relevantes o generan un gran impacto sobre el problema o sobre las posibles soluciones, tampoco se llegará a feliz término en las recomendaciones.

No se deberá descuidar la definición del plan de trabajo con su cronograma y sus responsables (muy necesarios para implementar las estrategias) de lo contrario las conclusiones no estarán ajustadas a la realidad y por ende no se podrán ofrecer soluciones integrales.

En el análisis DAFO se pueden incluir otros estudios complementarios como son el perfil de amenazas y oportunidades en el medio (POAM), el perfil competitivo (PC), el perfil de capacidades y fortalezas internas $(\mathrm{PCl})$, y si es posible, una base de referencia del medio (Benchmarking), que permiten presentar un panorama general del medio.

Además, no se deberá olvidar que la verdadera finalidad es la planificación estratégica e integrar procesos que anticipen o minimicen las amenazas del medio, el fortalecimiento de las debilidades, el potenciamiento de las fortalezas internas y el real aprovechamiento de las oportunidades. Dado que llegar solo hasta el diagnóstico (matriz DAFO) es solo quedarse a mitad de camino para tomar una decisión sin alternativas posibles, o con alternativas sin evaluar.

La estrategia es un modelo de cambio mediante la percepción del conjunto de pasos, acciones y vías que en un sistema posibilitan el tránsito del estado actual al futuro requerido y deseado (Ojeda 2008).

Para una adecuada orientación del proceso de desarrollo de la creatividad, se requiere del correspondiente planeamiento estratégico. Los tres elementos básicos a considerar para su formulación creativa son:

- Delimitar el estado de partida (o actual) del sujeto: barreras, condiciones facilitadoras, cualidades, dominio técnico que se posee.

- Interpretar los cambios deseados o esperados para un superior desarrollo de la creatividad.

- Exponer las diferentes estrategias en correspondencia con los dos aspectos anteriores.

- Tipos de estrategias 
Al combinar atributos del presente (fortalezas y debilidades) con otros del futuro (oportunidades y amenazas) se pueden determinar cuatro tipos de estrategias que podemos denominar estrategias combinatorias:

- Defensiva

- Ofensiva

- Supervivencia

- Adaptativa

En la formulación de estas estrategias es muy útil la técnica conocida como matriz DAFO:

Tabla 4. Matriz DAFO

\begin{tabular}{|c|c|c|c|}
\hline & Debilidades & Fortalezas & \\
\hline Amenazas & $\begin{array}{l}\text { Estrategia de } \\
\text { supervivencia }\end{array}$ & Estrategia defensiva & \multirow[t]{2}{*}{$\begin{array}{l}\text { Externo y } \\
\text { futuro }\end{array}$} \\
\hline Oportunidades & Estrategia adaptativa & Estrategia ofensiva & \\
\hline
\end{tabular}

Fuente: Ojeda, Jorge (2008)

\section{MATRIZ DAFO EN LA FORMULACIÓN DE ESTRATEGIAS}

Hay otros tres tipos de estrategias combinadas, pero utilizan elementos del presente, denominadas estrategias de mejora:

- Reactiva parcial

- Reactiva global

- Mantenimiento

Para ello también es útil la conocida como matriz DAFO añadida:

Tabla 5. DAFO añadida

\begin{tabular}{|c|c|c|c|}
\hline & Debilidades & Fortalezas & \\
\hline Fortalezas & Estrategia reactiva parcial & Estrategia de mantenimiento & Interno y \\
\hline Debilidades & Estrategia reactiva global & Estrategia reactiva parcial & \\
\hline
\end{tabular}

Fuente: Ojeda, Jorge (2008)

Las técnicas conocidas como matrices de cartera (entre ellas la denominada ELISA) ayudan a determinar algunas estrategias de:

- Inversión

- Desinversión

- Reconversión siguientes:

Otras estrategias suelen ser formuladas a partir del planeamiento circunstancial, como las

- Preventivas

- Cautelares

Finalmente, otras técnicas permiten formular estrategias de: 
Revista Geográfica Digital. IGUNNE. Facultad de Humanidades. UNNE. Año 9. № 18. Julio - Diciembre 2012. ISSN 1668-5180 Resistencia, Chaco

- Alianzas

- Relaciones

- Diferenciación

Más que una estrategia, por lo general, es necesario la formulación de varias estrategias de diferente tipo.

Así, hay barreras que existen y que quizás continuarán existiendo a pesar del esfuerzo por superarlas (algunas duran siglos) y no por ello será imposible realizar los intentos necesarios para superar los obstáculos. Esto se logra con una estrategia defensiva (fortaleciendo las actitudes para enfrentar las amenazantes barreras que perdurarán en el futuro).

Si se está en presencia de barreras posibles de ser superadas, la estrategia será ofensiva (determinar las acciones para eliminarlas o disminuir el impacto de los obstáculos).

En cambio si no se poseen las condiciones favorables para enfrentar ciertas barreras, ni se prevé que puedan ser superadas en el futuro, hacen falta estrategias de supervivencia.

En condiciones más favorables y, a pesar de no contar con las acciones requeridas, la estrategia puede ser adaptativa.

Cuando la situación es más favorable y lo que se trata de hacer es fortalecer las acciones para evitar mayores deterioros, la estrategia será de mantenimiento.

Si se tiene un conveniente proyecto para el desarrollo de las acciones que justifique los gastos requeridos, se desarrollará una estrategia de inversión.

En aquellos casos en los que no conviene continuar con gastos para superar los problemas, la estrategia será de desinversión o de reconversión.

Sin embargo, cuando se quieren evitar ciertas consecuencias para el futuro (como el surgimiento de nuevos o más poderosos obstáculos), se plantearán estrategias preventivas.

En algunas oportunidades, hay barreras que son difíciles de enfrentar y vencer, de allí que se requiera inteligencia, paciencia, además de ser muy cuidadosos para no echar a perder los logros alcanzados, entonces deben formularse estrategias cautelares a partir de una matriz de equilibrio de fuerzas con múltiples aplicaciones (Pardo Fernández y otros, 2005). De acuerdo con esta metodología, se listan las mismas a través de la aplicación de la técnica participativa Brainstorming o "lluvias de ideas".

Concluido el proceso se construye un eje de coordenadas en el que se muestran 4 cuadrantes. El valor del primer cuadrante es el resultado de la suma de todas las fortalezas con las oportunidades $(F+O)$. El valor del segundo cuadrante sería igual a la suma de las debilidades más las oportunidades $(D+O)$. El valor del tercero, resulta de la suma de las fortalezas más las amenazas $(F$ + A) y el cuarto se configura como resultado de la suma de las debilidades más las amenazas (D + A).

Por último se establece un gráfico que reúne los resultados a cabo a partir de la elaboración de una matriz de $2 \times 2$ que formula las estrategias más convenientes. En esta matriz DAFO las columnas establecen el análisis del entorno ( $1^{\mathrm{a}}$ columna: Amenazas, $2^{\mathrm{a}}$ columna: Oportunidades) y por filas el diagnóstico ( $1^{\mathrm{a}}$ fila: Puntos fuertes, $2^{\mathrm{a}}$ fila: Puntos débiles). Así se establecen 4 cuadrantes que reflejan las posibles estrategias a adoptar:

1. Estrategias defensivas

2. Estrategias ofensivas

3. Estrategias de supervivencia

4. Estrategias de reorientación 
Tabla 6. Matriz DAFO

\begin{tabular}{||c|c|c||}
\hline Matriz DAFO & AMENAZAS & OPORTUNIDADES \\
\hline PUNTOS FUERTES & $\begin{array}{r}\text { Estrategias } \\
\text { defensivas }\end{array}$ & $\begin{array}{c}\text { Estrategias } \\
\text { ofensivas }\end{array}$ \\
\hline \hline PUNTOS DÉBILES & $\begin{array}{l}\text { Estrategias de } \\
\text { supervivencia }\end{array}$ & $\begin{array}{c}\text { Estrategias } \\
\text { de reorientación }\end{array}$ \\
\hline \hline
\end{tabular}

Fuente: Pardo Fernández, Alfredo y otros (2005)

El proceso práctico de la matriz se concluye analizando aisladamente cada cuadrante. Es decir, para los Puntos Fuertes-Amenazas se tendrán que identificar cada uno de los puntos fuertes y cada una de las amenazas que posee del exterior. De esa forma cada intersección deberá ser analizada para estudiar las consecuencias y las acciones que de dicha situación puedan derivarse. Con esta información se podrá ir orientando la futura formulación de la estrategia.

1. Estrategias defensivas: se encuentra preparada para enfrentarse a las amenazas.

2. Estrategias ofensivas: es la posición en la que se aspira estar y se deberían adoptar estrategias de crecimiento.

3. Estrategias de supervivencia: consiste en enfrentar las amenazas externas sin las fortalezas internas necesarias para trabajar.

4. Estrategias de reorientación: se plantean las oportunidades que pueden aprovecharse, aunque se carece de la preparación adecuada. Se debería establecer un programa de acciones específicas y reorientar las estrategias anteriores.

Con estas herramientas se puede realizar el diagnóstico, teniendo en cuenta las peculiaridades de la actividad e información de cada sector.

Luego de realizar la valoración equilibrada de los aspectos claves del proyecto, se continúa con las correspondientes estrategias orientadas a potencializar las fortalezas y las oportunidades; a neutralizar, evitar o minimizar las debilidades y planear detalladamente las contingencias necesarias para enfrentar la materialización de las amenazas.

Tabla 7. Diagnósticos y estrategias

\begin{tabular}{|l||l||}
\hline \multirow{3}{*}{ PUNTOS FUERTES } & Capacidades y actividades clave. \\
& Recursos adecuados. \\
& Habilidades y recursos tecnológicos. \\
& Programa I+D. \\
\hline \hline \multirow{3}{*}{ PUNTOS DÉBILES } & No hay dirección estratégica clara. \\
& Incapacidad de aceptar los cambios necesarios en la \\
& estrategia. \\
& Atraso en I+D. \\
& Ingresos inferiores a la media. \\
& Infraestructura obsoleta. \\
& Sistemas ineficientes (exceso de problemas operativos). \\
\hline
\end{tabular}




\begin{tabular}{|l||l|}
\hline \multirow{3}{*}{ OPRTUNIDADES } & Entrar en nuevos mercados o segmentos. \\
& Ampliación de servicios para satisfacer nuevas \\
& necesidades. \\
& Crecimiento rápido del empleo. \\
& Disponibilidad de servicios asistenciales \\
\hline \hline \multirow{3}{*}{ AMENAZAS } & Instalaciones precarias. \\
& Deterioro ambiental. \\
& Crecimiento lento del empleo. \\
& Deserción escolar. \\
\hline
\end{tabular}

Fuente: Amaya Correa, Jailer (2004)

Las estrategias deben ser acciones objetivas, controlables, cuantificables y precisas para ejecutar; es decir, no deben propiciarse actividades genéricas o intangibles que puedan resultar incontrolables o imposibles de asegurar la cabalidad de las mismas (Amaya Correa 2004). De ese modo se debe elaborar una matriz de acciones y estrategias que se relacionen con cada una de las celdas de la matriz DAFO, las mismas se deben reunir en:

Estrategias y Acciones DO: En este grupo de acciones se deberán reunir los procedimientos que conducen a explicar cómo algunas debilidades se pueden considerar como oportunidades de mejoramiento o representan ajustes positivos.

Estrategias y Acciones DA: En este se deben reunir los planes orientados a cada una de las debilidades que actúan como amenazas. Se debe poner especial cuidado porque en este caso las debilidades ponen en riesgo las acciones del grupo.

Estrategias y Acciones FO: En este grupo se deben reunir los procedimientos conducentes a cómo las fortalezas internas o externas actúan como oportunidades que tienen el grupo para potencializar y asegurar el éxito de las acciones. Se deberán presentar acciones que permitan aprovechar al máximo las fortalezas.

Estrategias y Acciones FA: En este caso se deben agrupar los planes encaminados a cómo las fortalezas generalmente externas, pueden poner en riesgo el éxito de las acciones. Como se trata de acciones de prioridad muy alta, deben someterse a planes detallados que contengan o minimicen los efectos negativos que las amenazan.

La exposición de la matriz de estrategias debe incluir el cronograma y el análisis de las acciones a realizar. También se deben incluir los costos de cada una de las actividades.

El compendio final del análisis DAFO comprende:

La matriz de DAFO de diagnóstico con el detalle de todas las amenazas, fortalezas y debilidades de las acciones y su entorno.

La matriz de acciones para potencializar fortalezas y controlar o minimizar los riesgos.

El análisis de vulnerabilidad del grupo con sus correspondientes probabilidades de ocurrencia.

El análisis de riesgo de las acciones.

La estructura de responsabilidades para la ejecución de las actividades prioritarias a realizar.

Presupuesto necesario para cada una de las actividades propuestas.

Indicadores de Gestión para realizar el seguimiento a las diferentes actividades y su periodicidad (Amaya Correa, 2004, p.8). 


\section{CONCLUSIONES}

El método DAFO representa una herramienta de análisis y diagnóstico de vulnerabilidad si se obtiene solamente la conformación de una matriz de acciones con el análisis de riesgo, pero si es examinado en su globalidad, se logrará un modelo completo para la estructuración de proyectos y de planificación estratégica.

Con este modelo se logra tener una visión panorámica del problema, aunque conocer el modelo completo lleva a saber el potencial real del método (según la importancia del problema tratado) y definir hasta qué punto se avanza en el análisis, pues a medida que se adelanta en el proceso se conocen más detalles del proyecto y su entorno. (Amaya Correa 2004)

\section{BIBLIOGRAFÍA}

AMAYA CORREA, JAILER (2004) El método DOFA, un método muy utilizado para diagnóstico de vulnerabilidad y planeación estratégica? Neiva (Colombia), Universidad Nacional de Colombia.

DAVID, FRED R. (1997) Conceptos de Administración Estratégica, México, Editorial Prentice Hall.

DYSON, ROBERT G. (2004) Strategic development and SWOT analysis at the university of Warwick, Warwick Business School, University of Warwick, Coventry CV4 7AL, UK, European Journal of Operational Research 152, 631-640

IBARRA BENLLOCH, P. (1993) Una propuesta metodológica para el estudio del paisaje integrado. En Geographicalia 30. Revista del Departamento de Geografía y Ordenación del Territorio. Universidad de Zaragoza. España. Págs. 229-242.

OJEDA, JORGE (2008) Enfoques, evaluación, estrategias. Santiago de Cuba, Editorial Inspiración.

PARDO FERNÁNDEZ, Alfredo Dr.C.; Lic. Jacinto Duverger Goyanes; M. Sc. Gladys Ivette Maynard Bermúdez; M. Sc. Alfredo de la A. Izquierdo Hernández; Dr. Juan Carlos Rojas Fernández; Lic. Cesáreo Monteserín Puig Y Lic. Yuslay Ponce Sánchez. (2005) Matriz de balance de fuerzas innovada. BAFI. Premio Salud Pública, Venezuela.

ROMA PUJADAS y JAUME FONT (1998) Ordenación y Planificación Territorial. Espacios y Sociedades. Serie Mayor. (Madrid) España, Editorial Síntesis. 